\title{
LA DIALÉCTICA OPRESOR - OPRIMIDO EN LA CONSTRUCCIÓN DISCURSIVA DE LA VULNERABILIDAD DEL PEQUEÑO Y MEDIANO PRODUCTOR AGROPECUARIO DEL NORDESTE ARGENTINO
}

\section{THE DIALECTICS OPPRESSOR - OPPRESSED IN THE DISCURSIVE CONSTRUCTION OF THE VULNERABILITY OF THE SMALL AND MEDIUM AGRICULTURAL PRODUCER OF THE ARGENTINE NORTH-EAST}

\section{Mgtr. Emilas Darlene Carmen Lebus}

-Profesora Auxiliar de Primera Categoría Simple en "Introducción a la Geografía", Departamento de Geografía, Facultad de Humanidades, Universidad Nacional del Nordeste. -Profesora Titular de las cátedras de "Metodología de la Investigación" y "Taller de Tesis" en la Maestría en Derecho Procesal, Facultad de Derecho y Ciencias Sociales, de la Universidad Nacional de Rosario.

E-mail: emilaslebus@arnet.com.ar

\section{RESUMEN}

Este artículo se desprende de la investigación sobre "Vulnerabilidad socio-económica y semiótica del pequeño y mediano productor rural en el NEA", aprobada por la Secretaría General de Ciencia y Técnica de la UNNE, dirigida por Mgtr. Marta López y concluida en diciembre de 2010. Cabe destacar que este estudio tiene continuidad en la investigación en curso, titulada "Construcción discursiva del concepto de lo agrario y del escenario rural del NEA. Identidades y diferencias de un sistema complejo", bajo la misma Dirección e institución arriba mencionadas.

Este trabajo aborda específicamente cómo se construye la condición de oprimido entre los pequeños y medianos productores agropecuarios (PMP), en sus contextos territoriales y económicos concretos. Se busca identificar los mecanismos opresores que regulan la vulnerabilidad semiótica del PMP, en su dimensión constructiva, una de las facetas más ignoradas de su exclusión socioproductiva. El análisis del corpus sobre el tema pretende descubrir las contradicciones en los discursos desde la dialéctica del amo y del esclavo, basada en Hegel y ampliada desde el punto de vista de Paulo Freire.

\section{ABSTRACT}

This scientific paper parts with the investigation on "Social - economic and semiotic vulnerability of the small and medium rural producers in the NEA ", approved by the General Secretariat of Science and Technology of the UNNE, directed by Magister Marta Lopez and concluded in December, 2010. It is necessary to emphasize that this study has continuity in the investigation in process, titled "Discursive Construction of the concept of the agrarian thing and of the rural scene of the NEA. Identities and differences of a complex system ", under the same Direction and institution it arrives mentioned. This work approaches specifically how the oppressed's condition is constructed between the small and medium agricultural producers, in their territorial and economic concrete contexts.

It is sought to identify the oppressive mechanisms that regulate the semiotic vulnerability of the small and medium agricultural producer, in his constructive dimension, one of the facets most ignored of his social and productive exclusion. The analysis of the corpus on the topic tries to uncover the contradictions in the speeches from the dialectic of the owner and of the slave, based on Hegel and extended from Paulo Freire's point of view.

\section{PALABRAS CLAVES}

Vulnerabilidad socio-económica y semiótica; dialéctica, lógica de la opresión, autoconciencia, pequeños y medianos productores agropecuarios, categorías espaciales.

\section{KEY WORDS}

Social - economic and semiotic vulnerability; dialectic; logic of the oppression; self conscience; small and medium agricultural producers; space categories.

Publicado en formato digital: Mgtr. Emilas Darlene Carmen Lebus. LA DIALÉCTICA OPRESOR - OPRIMIDO EN LA CONSTRUCCIÓN DISCURSIVA DE LA VULNERABILIDAD DEL PEQUEÑO Y MEDIANO PRODUCTOR AGROPECUARIO DEL NORDESTE ARGENTINO. Revista Geográfica Digital. IGUNNE. Facultad de Humanidades. UNNE. Año 9. No 18. Julio - Diciembre 2012. ISSN 1668-5180 Resistencia, Chaco. En: http://hum.unne.edu.ar/revistas/geoweb/default.htm 
Revista Geográfica Digital. IGUNNE. Facultad de Humanidades. UNNE. Año 9. № 18. Julio - Diciembre 2012. ISSN 1668-5180 Resistencia, Chaco

\section{INTRODUCCIÓN}

En el desarrollo de este trabajo se presenta, en primer lugar, el método empleado en el análisis discursivo de los mensajes de PMP y su tratamiento conforme al marco conceptual adoptado, sustentado en el pensamiento dialéctico. El abordaje parte del enfoque hegeliano que examina la situación de opresión como proceso de liberación de la conciencia para que se constituya como autoconciencia. $Y$ dado que dicho planteo es bastante conocido (dialéctica del amo y el siervo en Hegel; o relación opresor-oprimido en Paulo Freire), se ha adoptado como estrategia operatoria presentar los discursos de productores y su análisis en un primer momento del trabajo, y, luego, introducir los conceptos en el proceso mismo de interpretación de los resultados emergentes.

Cabe aclarar que parte de los resultados del estudio citado fueron dados a conocer en las " $\mathrm{VI}$ Jornadas de Investigación y Debate: Territorio, Poder e Identidad en el Agro Argentino", desarrolladas en el Instituto de Investigaciones Geohistóricas (IIGHI), Resistencia, Chaco, en el año 2009.

\section{1.- MÉTODO Y CORPUS DE LA INVESTIGACIÓN}

Las estrategias aplicadas en este trabajo se apoyan en el método general que sostiene la investigación precitada, esto es, el análisis del discurso y la identificación de los elementos ontológicos que definen la relación sígnica, esto es, el término-objeto (objeto referido), el representamen (significante que alude al objeto representado) y el interpretante (en tanto "Regla" de producción de sentido que sostiene la relación semiótica). Estos tres componentes definen la concepción de signo según Peirce, a la cual adherimos, los cuales aparecen implícitos en los textos que integran el corpus de la investigación.

El análisis del discurso pretende incorporar los conceptos del enfoque arriba puntualizado, desde la perspectiva "dialéctica" como fundamento teórico y epistemológico del abordaje de este trabajo. Dichas ideas constituyen un marco de referencia conceptual consistente y adecuado para analizar la realidad de los PMP agropecuarios del NEA, eje temático de esta investigación. Este enfoque tiene gran potencia heurística para orientar el análisis de las situaciones vividas por estos agentes productivos desde el punto de vista semiótico (o sea, anclando en el plano de los discursos), ya que las categorías de análisis son generales y de amplio alcance, lo que las hace apropiadas al tema de indagación.

El "corpus" de investigación para este trabajo, en particular, se conformó con discursos construidos por los PMP agrupados en el Movimiento de Productores Autoconvocados (MPA), enfocando especialmente la realidad de los PMP del Norte santafesino en el contexto de la región geográfica del Nordeste Argentino (NEA). Cabe aclarar que el MPA tomó fuerza y protagonismo con motivo del conflicto del sector agrario argentino con el gobierno nacional, desarrollado en el primer semestre del 2008.

La necesidad de enfocar el análisis en los textos y otras formas de semiosis producidas por los PMP en el marco del MPA radica en la "singularidad" que asume dicho movimiento, que se define a partir de la diversidad que alberga (en cuanto a las características de los productores) y la autonomía de decisiones (en el plano semiótico), lo que -a mi criterio- proporciona las claves de comprensión de las nuevas formas identitarias, de representación social y de semiosis desarrolladas por los PMP, los que comienzan a revelar su vulnerabilidad discursiva que hasta hace poco tiempo ha estado al margen de los mecanismos hegemónicos de la comunicación social.

Con este criterio "operativo" se han seleccionado los materiales empíricos de análisis procedentes de las siguientes fuentes: 1- Circulación de mensajes entre los PMP a través de las cadenas de e-mail del movimiento mencionado (MPA). 2- Participación en reuniones de dicho movimiento (MPA), empleando "observación participante". 3- Noticias y mensajes de distintos dirigentes publicados en diarios, tanto las versiones electrónicas como en papel. 4- Puntos de vista y debates sobre el tema en diferentes programas televisivos y radiales.

Se han seleccionado más de 30 discursos, amén de muchos otros que fueron cotejados y analizados concomitantemente por su redundancia -en términos de pautas semióticas- respecto a los escogidos, Cabe recordar que aunque el análisis es "cualitativo", la cantidad incluida en la muestra asegura la variabilidad de los mensajes, condición necesaria para garantizar la confiabilidad de las ideas-síntesis construidas a partir del análisis e interpretación de los discursos.

Publicado en formato digital: Mgtr. Emilas Darlene Carmen Lebus. LA DIALÉCTICA OPRESOR - OPRIMIDO EN LA CONSTRUCCIÓN DISCURSIVA DE LA VULNERABILIDAD DEL PEQUEÑO Y MEDIANO PRODUCTOR AGROPECUARIO DEL NORDESTE ARGENTINO. Revista Geográfica Digital. IGUNNE. Facultad de Humanidades. UNNE. Año 9. No 18. Julio - Diciembre 2012. ISSN 1668-5180 Resistencia, Chaco. En: http://hum.unne.edu.ar/revistas/geoweb/default.htm 
Revista Geográfica Digital. IGUNNE. Facultad de Humanidades. UNNE. Año 9. № 18. Julio - Diciembre 2012. ISSN 1668-5180 Resistencia, Chaco

\section{2.- TRATAMIENTO Y ANÁLISIS DE LA TRAMA DISCURSIVA}

En principio cabe aclarar que el abordaje de los discursos es enfocado desde la perspectiva del $P M P$, o sea, priorizando el abordaje desde ese actor social y no desde la postura gubernamental, aunque ésta será considerada para remarcar la dialéctica que tiene lugar. Las expresiones vertidas por los emisores, incorporadas como ejemplo, aparecen encomilladas y en cursiva.

Las situaciones de opresión son expresadas por los PMP mediante enunciaciones que intervienen como "analogías" para dar cuenta de la realidad. Así, por ejemplo, la comparación con el Sistema Feudal resulta una metáfora muy propicia para presentar el "caso" en cuestión. Una metáfora no es la realidad, pero tiene "valor anticipatorio" para develarla, al abrir el camino al conocimiento.

En este sentido, se recurre a la metáfora de los pactos de vasallaje, propios del Medioevo, para aludir a la relación "gobierno - productores agropecuarios". Se señala que estos pactos estaban destinados a un Marqués, que juraba lealtad y sometimiento de su comarca al Rey, aceptando que éste fijara impuestos y decidiera sobre la vida de la gente en dicha comarca (sus súbditos o Vasallos). A cambio, el Rey le otorgaba protección y buen pasar.

Esta metáfora es utilizada para dar cuenta de las relaciones actuales entre el poder central (nacional) y los gobernadores e intendentes, refiriéndose, por ejemplo, al Decreto Presidencial de coparticipar una parte de las retenciones a la soja. Así, el Marqués vendría a ser aquellos gobernadores e intendentes "cooptados" por el poder "unitario" (centralista); los Vasallos (el pueblo, y específicamente, los productores agropecuarios que aportan sus tributos en términos de retenciones y otros impuestos). A cambio de la "concesión" gratuita que gobernadores e intendentes reciben (por vía del reintegro de una parte de las retenciones a la soja), éstos son "ungidos" por el Rey (poder central) escogiéndolos candidatos para las elecciones políticas, o "beneficiándolos" en el reparto de lo recaudado.

Bajo esta metáfora, el MPA de la provincia de Santa Fe envió una carta al gobernador respectivo, donde se le peticiona que no firme su aceptación de estos fondos co-participables provenientes de las retenciones al agro, rescatándose expresiones como éstas: "Ud. es gobernador de la provincia, no siervo feudatario..."; "Su compromiso es con los santafesinos y la Constitución Nacional Republicana y Federal, no con un sistema unitario y feudal..."; "Santa Fe está siendo <avasallada> de hecho por el poder central...; Ud. está a punto de suscribir una alianza que lo somete de derecho". La carta termina diciendo: "Póngase al frente de su provincia y lance el <grito federal> que ya lanzaron muchos de sus intendentes".

Esta última frase tiene un propósito contundente: el MPA de Santa Fe le está "implorando" al gobernador que se ponga del lado de la gente, de los productores, de los que trabajan, transmitiéndole un mensaje claro: que no se arrodille ante el poder central. En el fondo, subyace un profundo sentido "federal" y la referencia insoslayable al Interior productivo. Empero, el tiempo dejó al descubierto que la provincia aceptó dichos fondos co-participables provenientes del sector agrario, hecho que resultó incomprensible para los productores que "confiaban" en el gobernador.

La coparticipación de esta parte de lo recaudado por las retenciones a las exportaciones de soja es significada por los PMP como una forma "confiscatoria" de hacer recursos, inconstitucional, basada en un Decreto de Necesidad y Urgencia (DNU), contrario a las funciones del Ejecutivo, pues, según lo determina la Constitución, es el Congreso de la Nación quien tiene facultades para fijarlas. Sostienen que esa recaudación "debilita el aparato productivo de las provincias y fortalece al aparato clientelístico unitario".

Refiriéndose a los pactos de vasallaje, los mensajes indican que éstos consisten en celebrarse con el "dueño de la Kaja" (con K, aludiendo a la gestión de los Kirchner), y agregan: "Mientras tengamos intendentes y gobernadores mendigando a la Kaja rosada, tendremos un perfecto sistema feudal, cualquiera sean los candidatos". El deseo de liberarse de un sistema opresor remata en la siguiente idea: "No es la voluntad del Príncipe lo que transforma un sistema feudal en un sistema federal. Es la autonomía fiscal de las provincias y municipios".

En consecuencia, el sentido de los mensajes es comunicar la estrategia de "sometimiento": el gobierno central aparece en el lugar del "opresor" y los PMP en el de los oprimidos". La táctica del poder central reafirma este vínculo ya que aumenta el ejercicio de poder "real", socio-institucional, sobre los distritos administrativos de menor jerarquía (provincias y municipios) y aumenta también la vulnerabilidad sociogeográfica de las economías del Interior $\mathrm{y}$, por ende, del sistema productivo que involucra al PMP.

Publicado en formato digital: Mgtr. Emilas Darlene Carmen Lebus. LA DIALÉCTICA OPRESOR - OPRIMIDO EN LA CONSTRUCCIÓN DISCURSIVA DE LA VULNERABILIDAD DEL PEQUEÑO Y MEDIANO PRODUCTOR AGROPECUARIO DEL NORDESTE ARGENTINO. Revista Geográfica Digital. IGUNNE. Facultad de Humanidades. UNNE. Año 9. No 18. Julio - Diciembre 2012. ISSN 1668-5180 Resistencia, Chaco. En: http://hum.unne.edu.ar/revistas/geoweb/default.htm 
Revista Geográfica Digital. IGUNNE. Facultad de Humanidades. UNNE. Año 9. № 18. Julio - Diciembre 2012. ISSN 1668-5180 Resistencia, Chaco

Los discursos de los productores son elocuentes. En la carta antes mencionada, los productores autoconvocados piden al gobernador que actúe con ecuanimidad, en concordancia con el "principio" que ellos mismos levantan como bandera: no aceptar un sistema clientelístico y centralista que los autoconvocados rechazaron a partir del conflicto que "estalló" con la Res. 125, es decir, toda vez que se les ofrecieron compensaciones o subsidios fueron desestimados, por ser vistos como formas opresoras, que además no solucionan los problemas de fondo.

Reiteran el mensaje de apostar al trabajo para salir de la crisis, pero no para "sostener el barril sin fondo de gobiernos corruptos cuyas mentiras han salido a la luz". Por tanto, sí al trabajo y al esfuerzo, no al despilfarro ni a la corrupción; de esta manera expresan que apuestan a lo que saben hacer (conocimientos y cultura adquiridos de generación en generación).

Los discursos en general refuerzan la opresión que "sienten", es decir, la que los PMP experimentan en su realidad cotidiana. Describen esta realidad diciendo que "se" sienten "exprimidos", que están siendo "esquilmados" (por el gasto público que en los últimos años aumentó exageradamente -en un 60\%-). Expresan que se sienten "agobiados" por el peso que para ellos significa la suerte de la nación depositada en sus manos (dado que con los recursos sustraídos al sector, el gobierno financia políticas públicas), o por los destinos inciertos de la realidad social del país (metamensaje que viene desde el discurso opresor). Así surge la referencia a ciudades enteras que viven del empleo público. Señalan que el Estado, el Gobierno y los Políticos "necesitan límites, no más poder..."; que es preciso "recuperar el poder ciudadano, no cederlo"; que "este gobierno con el campo es autista".

Los mecanismos de opresión que atraviesan la política económica que se ha venido implementando en Argentina son reflejo del centralismo y concentración del poder, y están sustentados en una superestructura ideológica. Denotan, en esencia, un modelo de explotación que "somete a las regiones más alejadas y productivas del territorio a la expoliación del poder central". Es decir, subyace la voluntad de "salir" de esta situación y construir el país "federal". Las autonomías provinciales constituyen expresión de la voluntad ciudadana que clama por construir formaciones socioespaciales (formas productivas concretas, de base territorial) más equilibradas y acordes a la distribución regional de recursos y capacidades económicas.

Se señala, además, que no hay que caer ni en el liberalismo ni en el intervencionismo dogmático; que los productores no niegan la utilidad de muchas de las instituciones ligadas al agro, ni la necesidad de poner límites a la extranjerización de las tierras. Con ello dan a entender que el campo no es golpista, ni está a favor de la concentración económica, como se señala insistentemente desde los discursos gubernamentales.

Por el contrario, es "la situación" de los PMP la que ha llevado a la decisión inédita de agruparse en el MPA. Así, ellos mismos aclaran que casi nadie, entre los PMP, piensa en retenciones segmentadas o en recrear la Junta Nacional de Granos. Reclaman, en cambio, retenciones cero y apertura de las exportaciones agropecuarias. Las retenciones segmentadas son vistas como un anuncio intrascendente para la problemática que enfrentan los PMP, agravada por la sequía extrema que afecta, periódicamente, a varias comarcas del NEA (en particular, en el Norte santafesino, como en el Centro-Oeste chaqueño, la última gran sequía ha sido tan acuciante que hasta las lagunas se han secado por completo). Demás está decir que dichos ecosistemas, una vez expuestos a estos umbrales de extinción del fenómeno (en este caso, de los reservorios permanentes de agua dulce) difícilmente puedan volver a su estado habitual (como ha sucedido con las lagunas y esteros de los Bajos Submeridionales). No analizaremos, empero, en este momento los factores antrópicos que han coadyuvado, cuando no causado, estas transformaciones en ecosistemas frágiles.

El análisis de los discursos revela que el principal motivo por el cual los productores se han agrupado en el MPA es porque las instituciones agropecuarias tradicionales (las que existen) no dan respuesta a una necesidad primordial, que queda sintetizada en lo que ellos mismos manifiestan: "construir una agricultora con rostro humano". Enfatizan, y ¡hasta con tono enérgico y de enojo! que no se sienten "representados" por las instituciones tradicionales y que es preciso generar una que "represente" los intereses de los PMP específicamente.

Sin duda, éstos han venido operando desde los "bordes semióticos" (López, M., 2007; Magariños de Morentín, J., 2008) pues su vulnerabilidad discursiva se remonta, según afirmaciones de productores, a la época de las Juntas Nacionales (reguladoras de precios y de stocks de producción). Argumentan que el campo "ya era castigado" y no tenía ningún tipo de proteccionismo (como sí había, por ejemplo, para la industria). Esto denota que el problema se origina por un "abandono" a su

Publicado en formato digital: Mgtr. Emilas Darlene Carmen Lebus. LA DIALÉCTICA OPRESOR - OPRIMIDO EN LA CONSTRUCCIÓN DISCURSIVA DE LA VULNERABILIDAD DEL PEQUEÑO Y MEDIANO PRODUCTOR AGROPECUARIO DEL NORDESTE ARGENTINO. Revista Geográfica Digital. IGUNNE. Facultad de Humanidades. UNNE. Año 9. No 18. Julio - Diciembre 2012. ISSN 1668-5180 Resistencia, Chaco. En: http://hum.unne.edu.ar/revistas/geoweb/default.htm 
Revista Geográfica Digital. IGUNNE. Facultad de Humanidades. UNNE. Año 9. № 18. Julio - Diciembre 2012. ISSN 1668-5180 Resistencia, Chaco

propia suerte del primer eslabón de la cadena productiva. Frente a esta situación, consideran que ciertos acuerdos entre las entidades agropecuarias con el gobierno dejan al PMP en situación de mayor vulnerabilidad, por "desinformación" de los motivos que sustentan sus decisiones, incluso de la Mesa de Enlace.

\section{1.- Alcances "semióticos" de las expresiones de vulnerabilidad en los discursos de los productores}

Las expresiones que más se reiteran alertan contra distintas formas de engaño al productor agropecuario, como sucede con la propuesta de "bonificación"; los productores consideran que esto constituye una forma de legitimar un impuesto confiscatorio y de "discriminar" un sector de la producción "castigándolo" de este modo. Se llama permanentemente la atención sobre el carácter inconstitucional de los DNU. Si bien, los DNU están prohibidos para reglamentar cuestiones impositivas, en la práctica se legitima esta estrategia de opresión "de hecho".

Por otra parte, el MPA señala su desacuerdo con los "superpoderes" para manejar arbitrariamente la apertura y cierre de las exportaciones, lo que no sólo afecta a la comercialización de la producción agraria, sino que favorece la concentración del poder de decisión y de recursos en la fase exportadora (últimos eslabones de la cadena agroindustrial). Eso además significa fomentar, desde la misma ONCCA (Oficina Nacional de Control Comercial Agropecuario) la cartelización del mercado acopiador (por ejemplo, debido a estos mecanismos, de una retención establecida en $23 \%$ para el trigo, en la práctica se termina pagando por retenciones "reales" más del 40 \%).

Esta concentración en los últimos eslabones de la cadena perjudica enormemente al PMP al abrirse la brecha entre lo que éste percibe por su producción y el precio final del producto.

A criterio de los productores, el proyecto de ley que propone segmentar las retenciones y su disminución gradual no establece un horizonte claro de eliminación en un tiempo razonable. Por lo tanto, el PMP sigue en una situación de vulnerabilidad, en razón de que el mecanismo es el mismo: una especie de retenciones móviles avaladas por el Congreso, mientras el "contexto general" de comercialización prosigue y configura un manto de incertidumbre. Se establece así un doble mensaje: se manipula al destinatario para someterlo a la decisión de la parte más fuerte, es decir, cambia la forma pero el contenido es el mismo.

En relación a dicho proyecto, los productores sostienen que esto no se puede considerar una victoria política. Por el contrario, si, en el contexto del conflicto 2008, la Mesa de Enlace hubiera apoyado un proyecto de esta naturaleza, una vez obtenida la aprobación del Congreso, significaría una derrota política frente a la sociedad, porque eso implicaría que dentro de un breve período de tiempo los productores estuviesen reclamando nuevamente. Es decir, ellos advierten sobre la "aparente" solución de un proyecto así, pero que en realidad significa un ser inauténtico; no la solución verdadera, sino su "negación". Por lo tanto, la contradicción "intrínseca" persiste.

Sin embargo, el hecho de que los productores se den cuenta de estas "trampas" que encierran las propuestas ofrecidas como "aparente" solución a sus reclamos, constituye ya un primer paso para salir de la situación de opresión. Ya en el contexto del 2008, los productores advertían que se necesita también un "compromiso" genuino de la oposición con el reclamo de los productores, que debe darse "hoy" (refiriéndose al "período" del conflicto por la Res. 125), pues las promesas de que luego lo harán (según los resultados de las elecciones -se referían a las elecciones legislativas del 2009-) no se transformarán nunca en realidad. Refiriéndose al papel de los políticos opositores, señalaban en ese momento: "Hoy están a la caza de prosélitos, mañana estarán exigiendo el diezmo". Lamentablemente, el decurso de los acontecimientos, ligado a la alicaída semiosis de reclamo de los PMP, confirmó la sospecha que éstos tenían. Esta situación, sin duda, retroalimenta su vulnerabilidad no sólo porque los expone a la misma tendencia operante, sino, sobre todo, porque destruye su esperanza de cambio y su confianza en sí mismo para revertir su realidad.

Otra afirmación que permite intuir esta toma de conciencia de su situación es la siguiente: "El caballo de batalla de la política comunicacional del gobierno es decir: ¡Al campo no se lo conforma con nada!". En palabras de la Presidente de la Nación durante el mencionado conflicto: "No les importa quemar la patria con tal de salvarse". Puede constatarse aquí lo que sostiene Paulo Freire (2008), pues, en la lógica de la opresión, el que oprime busca hacer aparecer al oprimido en las antípodas de la sociedad. Y el que oprime no está dispuesto a ceder su poder, de ahí la dilación en resolver los problemas concretos, o bien la inacción frente a ellos. Se observa además el uso

Publicado en formato digital: Mgtr. Emilas Darlene Carmen Lebus. LA DIALÉCTICA OPRESOR - OPRIMIDO EN LA CONSTRUCCIÓN DISCURSIVA DE LA VULNERABILIDAD DEL PEQUEÑO Y MEDIANO PRODUCTOR AGROPECUARIO DEL NORDESTE ARGENTINO. Revista Geográfica Digital. IGUNNE. Facultad de Humanidades. UNNE. Año 9. No 18. Julio - Diciembre 2012. ISSN 1668-5180 Resistencia, Chaco. En: http://hum.unne.edu.ar/revistas/geoweb/default.htm 
Revista Geográfica Digital. IGUNNE. Facultad de Humanidades. UNNE. Año 9. № 18. Julio - Diciembre 2012. ISSN 1668-5180 Resistencia, Chaco

frecuente de la persuasión para confundir al destinatario del mensaje, y hasta podríamos afirmar, ¡para dejarlo en ridículo!

El MPA ha avanzado, durante el conflicto 2008 y hasta aproximadamente mediados del 2009, en este sentido de "descubrimiento" de las diferentes situaciones de opresión a que están sometidos, sobre todo los PMP, lo que constituye un proceso de semiosis que amerita ser analizado seriamente. Plantean que es preciso cambiar esta realidad para vivir en un país donde "no se discrimine a la actividad agropecuaria en relación a otras actividades"; y donde "no se discrimine al productor chico en relación al comerciante o industrial chico y donde no se discrimine al productor grande en relación al industrial o al comerciante grande"... "donde no se discrimine al Interior". Es evidente que estas expresiones constituyen el "inicio" de la toma de conciencia del problema de fondo: la diferenciación ante la ley y las formas anticonstitucionales de legitimar la opresión hacia el PMP y hacia el Interior del país.

Así expresan los autoconvocados: "Que la sociedad lo sepa es la humilde contrapartida que solicitamos para apoyar un proyecto que nos deja con las ganas... Pero que no queremos que nos deje sin banderas". Se constata así el esfuerzo de "reprimir" el deseo de salir "pronto" de esa situación, aunque esto signifique que pueda aumentar la "tenacidad" (bajo nuevas formas de semiosis más radicales, como las que se dieron en la primera parte del año 2008). Se "suprime" el deseo a favor de la "conservación" de un principio racional, lo que ya es un paso para avanzar hacia la superación de la situación. Ese paso es el comienzo del proceso de "concienciación".

\section{2.- ¿En qué medida las situaciones de opresión se reflejan en la conformación de la cadena de valor y las formas de semiosis vinculadas a ella?}

Las nuevas formas de semiosis aparecen, masivamente, en el contexto del conflicto 2008, como respuesta a las situaciones de opresión que "sienten" y "experimentan" los PMP, tales como: 1Convocatorias masivas" (reuniones de ciudadanos, dirigentes y agentes económicos del sector agroindustrial y comercial que apoyan al reclamo de los productores). 2- Presencia junto a las rutas (estado de alerta y movilización). 3- Petitorios presentados a autoridades políticas (aparece un reclamo fuerte a los "legisladores", que antes no tenían mayor protagonismo, vinculando la figura del "Senador" con el Interior, con lo federal).

Estos procesos semióticos implican no sólo el recurso de la palabra (forma de reclamo tradicional) sino nuevas estrategias expresivas, que comprometen el "cuerpo" y la "acción" manifestada en el compromiso de perseverar y hacer notar la presencia en los distintos eventos o convocatorias que se realizan. Aparece también el uso de los medios tecnológicos en la revolución de las comunicaciones (celulares y correo electrónico) que permiten la coordinación "horizontal" de las distintas formas de semiosis que despliegan en sus reclamos.

Esto permitió, por ejemplo, hacer conocer las situaciones de sequía extrema reciente que afectó a gran parte del NEA, en el período 2006 - 2010, aproximadamente, la más acentuada desde 195152 en que se dio una situación similar. Técnicos e investigadores que han estudiado el tema desde la agroclimatología afirmaron, en ese entonces, que "para los productores el panorama es desolador" y que de prolongarse la situación "el trigo no podría alcanzar siquiera para el consumo interno".

A raíz de la situación climática y la disminución del rendimiento de cultivos, los "contratistas" han bajado significativamente el porcentaje pautado con los productores por su función de "intermediarios". Esto hace pensar en lo opuesto, que cuando la situación climática es más favorable, el papel de los intermediarios en la cadena de valor significa un peso importante sobre el costo de producción del PMP. Así pues, la trama de los distintos agentes económicos que se articulan en torno a la producción agraria ha sido, y sigue siendo, en gran medida desconocida para el común de los ciudadanos.

Se dice, con frecuencia, que la actividad agropecuaria no genera empleo; sin embargo. Los productores argumentan que esta aseveración, reiterada por parte del gobierno, se funda en un profundo desconocimiento del sector. Tomando algunos datos para ilustrar, el Sindicato Argentino de Trabajadores Rurales y Estibadores (UATRE) es el que más afiliados tiene en el país, agrupando, actualmente, alrededor de 860.000 trabajadores inscriptos. Por otro lado, según el Censo de Población de 2001, ratificado por el Censo del 2010, sólo el 11\% de la población argentina es rural. Como sabemos, en nuestro país se considera urbana a toda población que habita en poblados de más de 2.000 habitantes. Sin embargo, con frecuencia, hay comunas que superar esta cantidad pero

Publicado en formato digital: Mgtr. Emilas Darlene Carmen Lebus. LA DIALÉCTICA OPRESOR - OPRIMIDO EN LA CONSTRUCCIÓN DISCURSIVA DE LA VULNERABILIDAD DEL PEQUEÑO Y MEDIANO PRODUCTOR AGROPECUARIO DEL NORDESTE ARGENTINO. Revista Geográfica Digital. IGUNNE. Facultad de Humanidades. UNNE. Año 9. No 18. Julio - Diciembre 2012. ISSN 1668-5180 Resistencia, Chaco. En: http://hum.unne.edu.ar/revistas/geoweb/default.htm 
Revista Geográfica Digital. IGUNNE. Facultad de Humanidades. UNNE. Año 9. No 18. Julio - Diciembre 2012. ISSN 1668-5180 Resistencia, Chaco

dependen casi totalmente del sector agropecuario (como el caso de Vera, en el Norte de Santa Fe), aunque para el INDEC son consideradas poblaciones urbanas.

Los productores aclaran, reiteradamente, la concatenación de actividades económicas que se articulan en torno a la producción agropecuaria, como las fábricas de maquinarias agrícolas, de autopartes, el trabajo de profesionales ligados al agro, el comercio, y el consumo en general en los pueblos, comunas y parajes distribuidos en la vasta geografía del Interior.

En consecuencia, si se considerara como población rural a la que habita en localidades de menos de 20.000 habitantes -dado su estrecha conexión con la producción agraria en el centro del territorio nacional y en el NEA- en la provincia de Santa Fe el 33 \% sería población rural, en Córdoba casi el $32 \%$ y en Entre Ríos el $40 \%$. Ese "ajuste estadístico" expresaría con mayor fidelidad la realidad de los pueblos del Interior que "viven del agro".

Por otro lado, si se tomara en cuenta que la cadena de valor generada por el agro no comprende sólo lo que se produce en el medio rural (como lo postula la tradicional categorización), y si se abandona la clasificación obsoleta de sector primario, secundario y terciario, por el concepto de "cadenas agroindustriales", deberíamos incorporar también otras actividades. ¿Por qué no se hace?

Como los productores lo expresan, esto sucede debido a la información incorrecta o desinformación (al decir de Watzlawick, 1994) sobre esas otras actividades que "complementan" y "hacen posible" que el campo argentino hoy esté entre uno de los más competitivos del mundo y con una muy alta rentabilidad y nivel de tecnología incorporada. En dichas cadenas habría que incluir a los veterinarios, los ingenieros agrónomos, los investigadores y extensionistas del INTA; sumar las industrias que procesan materia prima de origen agrario, y a las industrias que fabrican maquinarias agrícolas. Se estima que así tendríamos alrededor del $35 \%$ del empleo total del país generado por las cadenas agroindustriales, lo que significa un aporte del $40 \%$ al PBI nacional. Sin embargo, sobre estas cifras no hay acuerdo y algunos estudios consideran que estos valores son inferiores; la diferencia estaría en el método de clasificación de las actividades y su aporte, tanto en empleo como en valor de lo producido al PBI. Lo cierto es que la impronta del sector agroindustrial es altamente significativa y no se puede desconocer esta realidad, máxime en un país que depende del sector agrario como su base económica principal.

Por lo tanto, la falta de información veraz aumenta la vulnerabilidad de los productores, dejándolos expuestos a interpretaciones equívocas acerca de la actividad que desarrollan, porque al no ser valorizada la cadena que movilizan se genera otra forma de vulnerabilidad: la negación de su contribución a la sociedad como forma de exclusión. En los discursos oficialistas (no sólo de la Presidente, sino de sus más estrechos colaboradores) se refleja esta tendencia, señalándolos, peyorativamente, como aquellos individuos que sólo se preocupan de hacer plata para sí mismos. Se palpa aquí el efecto de distorsión de la información que termina ofuscando el papel de los productores en el entramado social, a pesar de ser los agentes productivos que intervienen en el origen de la cadena, que por dicha condición la sostienen. Su "rol social" queda totalmente solapado por la importancia concedida a la industria y a los servicios como sectores "independientes" (separados) de la producción agraria que es la base de la cadena.

La necesidad de incorporar una mirada integradora, y más realista, permitiría potenciar el diálogo social que es la condición ineludible para la concienciación. Empero, la negación a ver "otra realidad", impide que se construyan políticas más ajustadas a los hechos que permitan potenciar el valor de cada eslabón de las cadenas agroindustriales y sus efectos en la sociedad.

En los distintos mensajes de los productores se evidencia este planteo: la necesidad de hacer realidad políticas adecuadas y oportunas, en la inmediatez, pero apuntando al mediano y largo plazo. Esto exige, a la dirigencia toda, salir de un enfoque gubernamental centrado en la "tradición" para pasar a una "gestión inteligente" o -como también se denomina- "gestión distribuida" (que comparte el conocimiento como base de la gestión), lo que demanda a su vez un cambio en la concepción de la política y del poder: no un poder opresor sino un poder de liderazgo para liberar las situaciones de opresión y de vulnerabilidad de los agentes más débiles en la cadena productiva. Esto último requiere pasar de una política remedial y coyuntural a una proactiva y de planificación con visión de futuro, traducida en políticas de Estado. Para ello, la incorporación de conocimiento científico a la definición de tales políticas resulta clave.

Sin embargo, la continuidad de un modelo de poder verticalista, compartimentado entre los "sectores" de la producción, personalista y centralista, mitiga el poder de expresión de aquellos que forman parte de los niveles jerárquicos inferiores, como es el caso de los PMP. De ahí la condición de

Publicado en formato digital: Mgtr. Emilas Darlene Carmen Lebus. LA DIALÉCTICA OPRESOR - OPRIMIDO EN LA CONSTRUCCIÓN DISCURSIVA DE LA VULNERABILIDAD DEL PEQUEÑO Y MEDIANO PRODUCTOR AGROPECUARIO DEL NORDESTE ARGENTINO. Revista Geográfica Digital. IGUNNE. Facultad de Humanidades. UNNE. Año 9. No 18. Julio - Diciembre 2012. ISSN 1668-5180 Resistencia, Chaco. En: http://hum.unne.edu.ar/revistas/geoweb/default.htm 
"opresión" que experimentan, como emergente de mecanismos más profundos de política económica inadecuados para sus necesidades de producción en el actual contexto mundial. A nivel familiar y de pequeñas explotaciones agropecuarias, esto se traduce en mayor grado de vulnerabilidad socioeconómica y semiótica, pues en el fondo queda el amargo sabor de no ser escuchados por nadie, o bien, no encontrar el interlocutor válido.

Empero, la situación que afrontan los PMP es denunciada, como voces de apoyo, por muchos intendentes y presidentes de comunas que se suman a sus reclamos, destacando cómo las retenciones afectan al PMP y a los pueblos del Interior. En este plano, se suele comparar lo que aportan las provincias agrícolas en concepto de retenciones al Estado Nacional respecto a lo que recibirán éstas, como así también lo que recibirán cada una de las Comunas y Municipios por la coparticipación de las retenciones a la soja. Llevando esta comparación a valores per cápita, lo que un habitante de uno de estos pueblos agrícolas aporta y lo que recibirá en co-participación del Fondo Federal alberga una diferencia "abismal". Para la comuna de Chabás, por ejemplo, lo que recibirá es 417 veces menor de lo que aporta. O sea, "nada".

Esta realidad no sólo entraña una gran distorsión, sino que constituye otra manera de someter a aquellas comarcas que dependen de las cadenas de valor agroindustriales.

Por otra parte, si se comparan los valores históricos (de los últimos años) para provincias fuertemente agrícolas, cabe destacar que, por ejemplo, Santa Fe aportó más de 9.000 millones de dólares y recibió sólo 90 millones para atender la sequía que afectó a más de la mitad de la provincia, sobre todo el Norte, que a su vez es la región que tiene mayores desventajas estructurales y agroecológicas. Este dato ilustra la brecha entre lo que una provincia contribuye al Estado Nacional con relación a lo que percibe de ésta.

\section{3.- DIALÉCTICA DE LA OPRESIÓN}

"La realidad opresora, al constituirse casi como un mecanismo de absorción de los que en ella se encuentran, funciona como una fuerza de inmersión de las conciencias." (Freire; 2008:45)

Uno de los avances que han protagonizado los productores fue abrir el camino al conocimiento de la propia situación en la que están inmersos, es decir, el sistema de "opresión" como contexto totalizador. De años de silencio y de falta de medios adecuados para hacer oír su voz, los productores, en especial, los PMP, de las regiones más postergadas del país se han sumado al reclamo de aquellos que desarrollan sus actividades en la región más dinámica: el centro del territorio argentino (Pampa Húmeda).

Los reclamos existieron desde hace mucho tiempo atrás: reuniones con entidades del sector, con dirigentes políticos, en cooperativas, etc. Sin embargo, nunca hubo un reclamo generalizado, organizado y simultáneo como el que se desarrolló en el país desde marzo de 2008. Como lo han expresado los productores, la Res. 125 fue la "gota que rebalsó el vaso". Pero -siguiendo la metáfora- el vaso venía llenándose desde hace mucho tiempo con políticas desacertadas hacia el sector, no sólo desde la década del ' 90 , sino antes, que afectaron principalmente a las economías regionales que están sostenidas, por lo general, por PMP.

Dichas economías constituyen la base geoeconómica de muchas regiones y subregiones del Interior del país, sostenidas generalmente por una agricultura social, o de tipo familiar, que moviliza mano de obra y es multiplicadora de ingresos para las regiones al ser motor de las cadenas agroindustriales a que dan lugar.

En el Nordeste de Santa Fe esto puede afirmarse para la caña de azúcar y el algodón. Sin embargo, los cultivos "industriales" están decayendo desde la década del '80, provocando el retraimiento de las industrias, su re-localización (como ocurrió con la aceitera Vicentín), y el desplazamiento de población (migraciones) que incrementaron los problemas urbanos en las ciudades "intermedias" (caso de Reconquista y Avellaneda).

Es decir, en el Nordeste Santafesino y en gran parte de las comarcas productivas del NEA hay una "historia formativa" de las situaciones de opresión, ligada directamente a la vulnerabilidad de los productores más pequeños y más dependientes de cultivos "regionales" (generalmente monocultivos). Las formas que ella adopta son diversas: altos impuestos, insumos costosos a precio dólar, semilla fiscalizada, endeudamiento progresivo de los productores, obsolescencia de la maquinaria agrícola del PMP, y distanciamiento competitivo respecto de los grandes productores con

Publicado en formato digital: Mgtr. Emilas Darlene Carmen Lebus. LA DIALÉCTICA OPRESOR - OPRIMIDO EN LA CONSTRUCCIÓN DISCURSIVA DE LA VULNERABILIDAD DEL PEQUEÑO Y MEDIANO PRODUCTOR AGROPECUARIO DEL NORDESTE ARGENTINO. Revista Geográfica Digital. IGUNNE. Facultad de Humanidades. UNNE. Año 9. No 18. Julio - Diciembre 2012. ISSN 1668-5180 Resistencia, Chaco. En: http://hum.unne.edu.ar/revistas/geoweb/default.htm 
Revista Geográfica Digital. IGUNNE. Facultad de Humanidades. UNNE. Año 9. № 18. Julio - Diciembre 2012. ISSN 1668-5180 Resistencia, Chaco

mayor capacidad económica para tecnificarse; asimismo, excesiva burocracia fiscal y laboral (ya que estamos hablando de cultivos sociales).

Para el PMP esto fue definiendo su condición de "oprimido", sin voz ni voto, ya que la falta de poder para tomar decisiones que reviertan la situación significó, durante mucho tiempo, que ni siquiera fueran escuchados. Se priva así de lo más valioso que tiene el individuo, pues -como señala Freire (2008)- a través de la palabra el hombre se hace humano.

Por lo tanto, lo que se desnuda a partir de la Res. 125 es esta realidad de sometimiento y de "encierro" dentro de los campos que experimentaba el PMP. De ahí que se sintió reflejado en miles de situaciones análogas, reconociéndose en otros rostros "humanos" expresivos de situaciones similares, y, por eso, agrupándose en el MPA, salió a las rutas y fue el que sostuvo la postura más radical durante la etapa más dramática del conflicto (primer semestre de 2008).

Desde ese momento, los productores dejaron de estar en el anonimato y pasaron a ser actores concretos, identificados y "situados". Se develó que en nuestro país, y especialmente en el Norte, existe aún una agricultura hecha por hombres (no por máquinas), pero que está siendo "avasallada" por las formas sutiles en que avanza la concentración de tierras y la expansión de cultivos tecnológicos (como la soja) en vastas extensiones territoriales. Así, esos agricultores arraigados a sus lugares de origen están siendo heridos de muerte al tocarle lo más valioso que "aún" les queda, la tierra, cuando no la han perdido ya debido a su insolvencia económica, por embargos o quiebres de las cooperativas que los agrupaban.

Esta situación "extrema" de vulnerabilidad que venían soportando fue desnudada con el conflicto campo - gobierno. Las formas de semiosis que comenzaron a surgir a partir del detonante (Res. 125) son diversas: desde la distribución de folletos a la población civil que circula en las rutas, la elevación de cartas y petitorios a los representantes políticos (legisladores sobre todo), a las distintas formas de manifestación al lado de las rutas o de acción "corpórea" en éstas. La acción directa en los espacios públicos configuró la forma semiótica por excelencia durante el conflicto. Luego, tras pasar a otras semiosis ligadas a la intervención directa en la política (que ha conseguido algunos representantes del sector agrario en el Congreso Nacional), esas plurales y novedosas semiosis -por la extensión que han adquirido durante el conflicto-, quedaron casi en el olvido. Sobrevino entonces una etapa de silencio, de un silencio profundo que se presenta casi como una paradoja de la euforia y "vigilia" en las rutas de aquellos días del conflicto.

Entre los PMP existe un descreimiento muy generalizado acerca de las propuestas de cambio "prometidas" para el sector. Se reclama "sinceramiento" y soluciones "genuinas y de fondo": se dice, por ejemplo, que no quieren "parches" o "maquillajes" por considerar que esto constituye una "dilación sin sentido". Estas afirmaciones son una manera de hacer tomar conciencia sobre el problema. No aceptar soluciones parciales o propuestas que sólo permanecerán en el plano del discurso permite a los productores descubrirse a sí mismos y, a la vez, identificarse ante la sociedad.

Se señala, recurrentemente, la necesidad de "mantenerse unidos". Esta construcción de lo "Uno" (categoría de la Unidad) por encima de las individualidades constituye una estrategia que pugna por salir del estado de opresión. Implica forjar el camino de la "autoconciencia", pero para lograrlo se remarca también que es preciso "mantener la calma", "no perder los estribos" para poder avanzar. Es decir, que hay un proceso de autoconocimiento que permitió a los productores re-conocer la situación de opresión en la que vienen operando y, al mismo tiempo, conocerse a ellos míos, salir del en-sí para re-encontrarse en-otros. La captación de la "diferencia" en el otro implica "negar" el estado inicial (la opresión en su estado original que se desarrollaba en el anonimato total, que es un estado de desconocimiento de lo que pasa).

En esta fase, las distintas formas de semiosis constituyen procesos de resiliencia, como lo manifiestan los mismos productores: "es una resistencia civil a la opresión".

\section{1.- ¿Cuál es la interpretación de esta realidad?}

Posicionándonos en el marco conceptual de la investigación, la dialéctica que Hegel plantea entre el Amo y el Esclavo, así como la dialéctica opresor - oprimido que aborda Freire, aportan un principio de comprensión del estado de situación que define la relación entre los PMP y el contexto (político-gubernamental) en que se desarrolla el conflicto.

Dice Hegel: "La conciencia es en y para sí en cuanto que y porque es en sí y para sí para otra autoconciencia; es decir, sólo es en cuanto se la reconoce." (2002:113)

Publicado en formato digital: Mgtr. Emilas Darlene Carmen Lebus. LA DIALÉCTICA OPRESOR - OPRIMIDO EN LA CONSTRUCCIÓN DISCURSIVA DE LA VULNERABILIDAD DEL PEQUEÑO Y MEDIANO PRODUCTOR AGROPECUARIO DEL NORDESTE ARGENTINO. Revista Geográfica Digital. IGUNNE. Facultad de Humanidades. UNNE. Año 9. No 18. Julio - Diciembre 2012. ISSN 1668-5180 Resistencia, Chaco. En: http://hum.unne.edu.ar/revistas/geoweb/default.htm 
Esto significa que el proceso de autoconciencia (tomar conciencia del ser de uno mismo) implica "necesariamente" la autoconciencia del otro (que el otro también tome conciencia de sí mismo). En este movimiento, Hegel plantea la dialéctica, por la cual, el primer momento que implica un desplazamiento a otro se encuentra "fuera de sí".

El proceso de liberación de una situación de opresión se inicia con alguien que, frente a algo (que en Hegel es el objeto de la apetencia) es puesto en situación, quien constituye el primer paso. En este caso, ese puntapié lo dieron los productores. Como dijimos, la Res. 125 fue la "oportunidad" que posibilitó la caída del velo, es decir, el "significante" que desencadenó un proceso semiótico. Ese primer momento es "fuera de sí" dado que el oprimido, al comenzar a intuir la realidad que lo oprime, tiende a expresar a viva voz su situación.

Esto se traduce con carácter de "sorpresa" para el otro, para la sociedad que mira desconcertada lo que pasa, sin comprender la esencia del problema. Para el opresor, esto se advierte como el primer síntoma de "alerta" ante un otro (oprimido) que está despertando. Así surgen mensajes de primera mano: "son golpistas, oligarcas, vende patria, quieren ganar demasiado...". Estos términos intentan enfatizar que el punto opuesto adonde ha ido el desplazamiento del sentido, por efecto semiótico, es erróneo, por lo que se busca volver al estado inicial (restituirlo al estado de opresión o de no-conciencia). Pero lo que no advierte el opresor es que por más que vuelva la calma, nunca se retorna al punto de partida. Para el productor, este estado de cosas (en torno a los dichos que se entrecruzan en ese movimiento de concienciación) tampoco es un retorno al estado inicial. Algo ha cambiado para ambos.

Surge entonces la autoconciencia "duplicada" (según Hegel). Por una parte, la autoconciencia se ha perdido en un primer momento porque se encuentra en otra esencia (desplazada), pero entonces tampoco ve a lo otro como esencia, ya que "se ve a sí misma en lo otro" (ibídem:113). Hegel dice que es preciso superar su ser otro, es decir, ese primer doble sentido, pero también debe superarse un segundo doble sentido: "superar la otra esencia independiente, para de este modo devenir certeza de sí como esencia... y (...) con ello superarse a sí misma, pues este otro es ella misma". (ibídem:114)

Este desplazamiento de la conciencia en un ser otro adquiere características de extrañeza, de lo ajeno, para ambas partes. De ahí la incomodidad que se siente en ese estado (siendo otro). Esto se evidenció cuando los productores se decían a sí mismos (al comienzo del conflicto en el 2008): "No sé qué hago yo en esta ruta"; "Nuestro lugar está en el campo, produciendo"; Y agregaban: "Pero, no nos queda otra".

Este proceso lo deben realizar las dos autoconciencias; cada una de ellas se representa como el hacer de una respecto de la otra, que es un hacer hacia sí como hacia otro. Se entabla entonces una lucha de las autoconciencias contrapuestas -siguiendo a Hegel- que se presentan como figuras independientes centradas en la determinabilidad (situación determinada) de cada una.

Cado uno se "reconoce a sí mismo" como "reflejado" en otro. El objeto referente en el proceso semiótico se halla desplazado en la acción del otro, y oficia como mecanismo de control de la propia acción. De ahí el cruce de expresiones de ambas partes y la "espera" de la acción recíproca, aguardando la respuesta de la otra. En este momento dialéctico se sitúa la dilación como trasfondo del problema.

En el hacer duplicado, antes mencionado, se entabla una dialéctica entre la vida y la muerte. ¿Por qué? Porque en el hacer del otro cada uno tiende a la muerte del otro; pero significa también un hacer por sí mismo, ya que lo anterior conlleva el riesgo de perder la propia vida. Hasta aquí, el proceso de constitución de la autoconciencia, como ser-para-sí, sólo se expresa como un desplazamiento del en-sí en para-otro.

Para que la conciencia devenga ser-para-sí como resultado del paso por la negatividad absoluta, de ambas autoconciencias, es preciso entablar una lucha de vida y muerte. En palabras de Hegel:

El comportamiento de las dos autoconciencias se halla determinado de tal modo que se comprueban por sí mismas y la una a la otra mediante la lucha a vida o muerte. Y deben entablar esta lucha, pues deben elevar la certeza de sí misma de ser para sí a la verdad en la otra y en ella misma. (Ibidem:116)

Éste es el momento de "máxima diferenciación" entre las partes. Cada una está encerrada en torno a sí, mostrando la determinabilidad que atraviesa "su" realidad cotidiana, pero que aún no es el alumbramiento de la verdad, sino apenas un centello de un fragmento de ésta.

Publicado en formato digital: Mgtr. Emilas Darlene Carmen Lebus. LA DIALÉCTICA OPRESOR - OPRIMIDO EN LA CONSTRUCCIÓN DISCURSIVA DE LA VULNERABILIDAD DEL PEQUEÑO Y MEDIANO PRODUCTOR AGROPECUARIO DEL NORDESTE ARGENTINO. Revista Geográfica Digital. IGUNNE. Facultad de Humanidades. UNNE. Año 9. No 18. Julio - Diciembre 2012. ISSN 1668-5180 Resistencia, Chaco. En: http://hum.unne.edu.ar/revistas/geoweb/default.htm 
En ese estado, la "percepción del problema" se sectariza, apareciendo cada postura como verdad única, pero que en realidad es falsa verdad. Freire (2008) dice que en esas circunstancias ellas están "ensimismadas", o sea, atrapadas en un ser inauténtico. La multiplicación de cortes de rutas por los productores (más de 90 en marzo de 2009; en el momento de máxima tensión en el 2008 fueron muchos más) denota este ensimismamiento en su propia singularidad (problema) sin tener en cuenta sus efectos en el resto de la sociedad. Por otra parte, el gobierno bombardeando a la opinión pública con múltiples "comunicados" intenta "hacer aparecer" al otro como perverso, autoritario (ejemplo: "¿quiénes son estos señores para arrogarse decidir sobre el destino de toda la nación?"), y se recurre a términos que intentan descalificar o desvirtuar la realidad (como: "tienen sus cuatro por cuatro y no se conforman") para mostrar una realidad que no existe para el PMP.

El oprimido puede pasar, en un primer momento, a ser opresor o sub-opresor. Esto es así porque su estructura de pensamiento está condicionada por la contradicción en la que vive cotidianamente. Por eso, ese primer momento es engañoso. Así sucede que el despojado de la tierra al convertirse en capataz tiende a reproducir el mismo patrón opresor que él experimentó.

Para salir de esa situación es preciso efectuar un reconocimiento crítico de la realidad en que se está inmerso. El problema de fondo -siguiendo a Freire- es que la dialéctica opresor - oprimido radica en la propia conciencia del oprimido. Esto se traduce en la contradicción entre el ser-en-sí (o falso ser-para-sí) y el ser-para-otro. Cuando el oprimido descubre su condición de opresión, tiende a negar si estado inicial, desplazándose al polo opuesto, donde se constituye como ser-para-otro. De ahí que termina identificándose con la conciencia opresora y puede adoptar conductas del opresor.

Las formas de semiosis lo expresan. Por ejemplo, cuando los productores estuvieron a punto de radicalizar el conflicto y tomaron injerencia "directa" en el problema, empleando, a su vez, discursos de descalificación del otro (opresor), o bien, tomando partido en una acción concreta (por ej. ganar la "pulseada" en el Congreso) o cortar rutas poniéndose en calidad de policías: "este pasa, este no". Aparecen juicios muy duros cuando el otro no actúa como el oprimido deseara, acusando de "traidores a la provincia" a los diputados que por "obediencia debida" al gobierno nacional le votan todos los proyectos. Es decir, se enfatiza el lado "negativo" desnudando al otro, lo cual no es necesariamente malo siempre que se lo conciba en un proceso de concienciación mutua para salir del estado de situación que oprime al Interior del país, a las economías regionales y a los productores más vulnerables.

Por otra parte, el opresor teme perder su poder para oprimir. Así aparecieron, en el 2008, conductas de atropello para impedir manifestaciones de los productores, como cuando algunos camioneros fueron enviados por dirigentes para frustrarlas (poniéndose en el lugar de "gendarmes"), o bien con expresiones amenazadoras y de descalificación. Un dirigente gremial expresó, en ese momento: "No existen" (refiriéndose a los productores), con lo cual se busca "minimizar" la fuerza del oponente para ubicarlo en el lugar de la nada. Y agregó: "Los camioneros los van a cagar a trompadas. Esperá unas horas... la orden está dada." Se desliza el conflicto al nivel de la fuerza bruta, hacia acciones de "aparente" irracionalidad, aunque en el fondo son coherentes con la razón y que puede expresarse así: iseguir manteniendo las riendas sobre el oprimido! Más aún, los productores han advertido sobre la estrategia de "provocación" para que el conflicto se radicalice.

La realidad opresora funciona como una especie de atractor extraño, en torno al cual pivotea la vida del oprimido. De ahí que esa realidad se exprese -según Freire- como fuerza "domesticadora". Es decir, acostumbramiento a la situación de sometimiento. Por ello el primer paso para salir de esa situación consiste en "descubrir" claramente al opresor, lo que supone superar la complicidad con la realidad opresora que vive. En el fondo, es este descubrir del sistema opresor lo que está en juego.

Hegel se refiere a la radicalización del proceso de formación de la autoconciencia. En sus palabras: "El individuo que no ha arriesgado la vida puede sin duda ser reconocido como persona, pero no ha alcanzado la verdad de este reconocimiento como autoconciencia independiente." (Ibidem:116). En ese momento: "Se halla fuera de sí y tiene que superar su ser fuera de sí; el otro es la conciencia entorpecida de múltiples modos y que es; y tiene que intuir su ser otro como puro ser para sí o como negación absoluta." (ibídem:116).

¿Cómo se traduce en el plano del discurso este salirse fuera de sí, en el caso de los PMP que venimos abordando? El primer momento constituye un "anoticiamiento" hacia la sociedad. Si bien ésta ve lo que pasa, lo "registra" como un hecho más, no alcanza a comprender los motivos de los PMP, en su lucha existencial por su condición de oprimido.

Publicado en formato digital: Mgtr. Emilas Darlene Carmen Lebus. LA DIALÉCTICA OPRESOR - OPRIMIDO EN LA CONSTRUCCIÓN DISCURSIVA DE LA VULNERABILIDAD DEL PEQUEÑO Y MEDIANO PRODUCTOR AGROPECUARIO DEL NORDESTE ARGENTINO. Revista Geográfica Digital. IGUNNE. Facultad de Humanidades. UNNE. Año 9. No 18. Julio - Diciembre 2012. ISSN 1668-5180 Resistencia, Chaco. En: http://hum.unne.edu.ar/revistas/geoweb/default.htm 
Revista Geográfica Digital. IGUNNE. Facultad de Humanidades. UNNE. Año 9. No 18. Julio - Diciembre 2012. ISSN 1668-5180 Resistencia, Chaco

Sin embargo, el mensaje de los productores es preciso, aunque muy sugestivo. Por ejemplo: "Pedimos que nos saquen la pata de encima"; "Pedimos que nos dejen producir". Son los propios productores quienes recuerdan a otros productores que es preciso mantenerse en el eje del reclamo, advirtiendo a sus pares: "Pedir soluciones a un tercero es poner nuestra vida en sus manos"; "Nadie pide soluciones a un chorro que lo está asaltando"; "Nadie le pide soluciones a quien pagó la mitad de su trabajo"; "Cada vez que pedimos soluciones o ayuda estamos dando un mensaje negativo y un <metamensaje> más negativo aún". Es decir, estas expresiones apuntan a descubrir cuál es el trasfondo del problema: la opresión y no el opresor de turno.

Este proceso implica que cada una de las conciencias que participan en esa dialéctica se desintegre en las determinabilidades contrapuestas. El término medio es, en ese momento, al decir de Hegel, "una unidad muerta, que se desintegra en extremos muertos, que simplemente son o no son contrapuestos". Y guardan "el uno con respecto al otro la libertad de la indiferencia, como cosas". (ibídem:117). Este estado de contradicción absoluta (recaída como coseidad) es el momento de la negación abstracta; no es aún la negación de la conciencia. Es entonces un estado intermedio en el movimiento dialéctico, y la negación del otro se presenta bajo dos figuras contrapuestas (o como conciencia en la figura de la coseidad), no como conciencia independiente. Termina Hegel diciendo: “... una es la conciencia independiente que tiene por esencia el ser para sí, otra la conciencia dependiente, cuya esencia es la vida o el ser para otro; la primera es el señor, la segunda el siervo. (ibídem:117)

Existe un miedo latente en ambos de asumir un nuevo estado frente a la situación de opresión: el oprimido teme "asumir" la libertad y el opresor teme "perder" la libertad de oprimir. Esto significa que el miedo es el síntoma expresivo de la situación opresora, que mantiene "presos", en última instancia, tanto al opresor como al oprimido.

El proceso de concienciación no sería posible sin el re-conocimiento de cada conciencia en otra. El decir de Freire (2008), el hombre se libera en "comunidad". De ahí que tanto el opresor como el oprimido tiendan a comunicar su situación, buscando apoyo en otros, aspecto éste ineludible en el movimiento de construcción de la autoconciencia que opera como proceso de liberación (o salida de la contradicción). Las dificultades que emergen en este proceso de semiosis en el marco de la comunidad (o en el plano de los co-sujetos) conlleva la "duda" respecto de si proseguir en el camino de la liberación, o bien, permanecer en la misma situación, lo que significa una "duda existencial" entre el compromiso y la acción, por un lado, y el estatus quo y el conformismo, por otro.

Este comportamiento dual se manifiesta no sólo en los PMP sino también en aquellos que "dicen" apoyar a éstos. Así, algunos intendentes que participaron en las reuniones del MPA de Reconquista, expresaban a viva voz su adhesión al reclamo de los productores, pero luego terminaron aceptando la coparticipación de las retenciones a la soja (apoyados incluso por entidades locales vinculadas al sector agrario), o, lo que aún es más perverso, apoyaron en las Legislaturas Provinciales los aumentos siderales de los impuestos a la tierra rural, aún a sabiendas de la situación por la que atraviesan los PMP, cuya realidad es muy distinta de los pooles de siembra y de quienes detentan grandes extensiones latifundistas. De ahí que los PMP no encuentren ni en las entidades rurales tradicionales, ni en los dirigentes políticos, el sentido pleno y genuino de la representación que aquéllos "dicen" encarnar.

¿Cuál es la verdadera situación de "opresión" que envuelve tanto al oprimido como al opresor? Podríamos expresarla así: 1- Pervivencia de un modelo territorial y económico de un país fuertemente "centralista"; 2- Incremento de la concentración de poder económico en unos pocos actores mientras la gran mayoría de los agentes productivos (que son PMP) afrontan una pérdida creciente de rentabilidad y competitividad, con lo cual tienden a "salirse" del tejido productivo; 3- Actividad parasitaria del Estado (expresada en distintas formas: burocracia, gastos superfluos, excesivo empleo improductivo); 4- Manejo discrecional de fondos públicos para fines no productivos (campañas electorales o clientelismo político); 5- Desinversión progresiva en las estructuras públicas elementales y desatención de las funciones básicas del Estado (salud, educación, seguridad y defensa); 6Abandono del Interior productivo a su propia suerte, sin inversión en infraestructura básica para el desarrollo; 7- Postergación en la búsqueda de soluciones a los problemas diversos de las economías regionales, que son el fundamento del entramado social de los pueblos y del arraigo de la población a sus lugares de origen; 8- Imprevisibilidad de las acciones económicas a futuro (gran incertidumbre); 9- Ausencia de políticas de Estado que atiendan tanto a la base productiva nacional como al desarrollo social de una manera integrada; 10- Falta de conocimiento sobre la realidad de las

Publicado en formato digital: Mgtr. Emilas Darlene Carmen Lebus. LA DIALÉCTICA OPRESOR - OPRIMIDO EN LA CONSTRUCCIÓN DISCURSIVA DE LA VULNERABILIDAD DEL PEQUEÑO Y MEDIANO PRODUCTOR AGROPECUARIO DEL NORDESTE ARGENTINO. Revista Geográfica Digital. IGUNNE. Facultad de Humanidades. UNNE. Año 9. No 18. Julio - Diciembre 2012. ISSN 1668-5180 Resistencia, Chaco. En: http://hum.unne.edu.ar/revistas/geoweb/default.htm 
Revista Geográfica Digital. IGUNNE. Facultad de Humanidades. UNNE. Año 9. № 18. Julio - Diciembre 2012. ISSN 1668-5180 Resistencia, Chaco

regiones geográficas argentinas y de los modos de vida de la población del "Interior Profundo", especialmente del NEA y NOA.

Estos aspectos determinan las contradicciones que marcan el estado actual de la dialéctica entre los productores y el gobierno, en la cual, unos y otros tienden a re-producir conductas duales que se deslizan desde la apuesta al cambio o el permanecer en la situación de opresión, ya que para salir de ello es necesario el pensamiento crítico, la intención de descubrir el problema de fondo, realizar el diálogo sincero y dar el tiempo necesario para efectuar un movimiento de reflexión, de debate crítico, apostando al esfuerzo de superación de la situación.

\section{4.- CATEGORÍAS ESPACIALES IMPLÍCITAS EN LA DECONSTRUCCIÓN SEMIÓTICA DE LA VULNERABILIDAD}

¿Qué ha permitido revelar el proceso discursivo instalado por los PMP, sobre todo a partir del conflicto que se ha suscitado en el 2008, pero cuyos antecedentes vienen de hace más de 30 años, en lo que respecta a la dimensión geográfica del problema? Es decir, ¿qué categorías cognitivas, inherentes al espacio geográfico, son puestas de manifiesto?

Una categoría es una noción genérica que posibilita construir el conocimiento. En tal sentido, es un constructo, un concepto, que posibilita pensar, analizar e interpretar la realidad empírica.

Las categorías espaciales, que pueden ser significadas desde el marco interpretativo de la dialéctica de la opresión, se manifiestan como fenómeno semiótico generado desde la propia vivencia del productor, de su "estar ahí", en un medio geográfico determinado, donde las relaciones opresor oprimido se realizan y actualizan permanentemente. Dichas categorías están profundamente "entrañadas" en las representaciones mentales que el productor construye en situaciones de vulnerabilidad, ligadas tanto a su capacidad de expresión como a las formaciones socioespaciales donde opera.

La relación de opresión, abordada en este trabajo, puede ser vista como un proceso cognitivo, donde no sólo aflora el problema en sí, sino también las categorías que sostienen los discursos, que se revelan, en el plano de la existencia, como semiosis. Me interesa destacar, en particular, las categorías "espaciales", o sea, aquéllas que sostienen la perspectiva geográfica del problema. Desde este punto de vista, los discursos dan cuenta de las siguientes categorías:

-Región geográfica: aparece identificada también como Interior productivo, aunque este último puede significar, asimismo, el "espacio periférico" concreto que constituye el escenario de referencia para el productor. De este modo, se subrayan los procesos de periferia que afectan al territorio extrapampeano y que se evidencian tanto en la carencia de infraestructura como en el débil poder socioeconómico y semiótico- de los agentes productivos que operan en esta Geografía del Interior.

-Economías regionales: si bien no es una categoría espacial en sentido estricto, sí aparece el referente "territorial" para singularizar el tipo de producción que se desarrolla en el Interior del país, basada en la especialización productiva en cultivos agroindustriales que movilizan las cadenas de valor.

-Territorio: aparece en reiteradas oportunidades, en las cuales el significado se asocia con el profundo "sentido" geográfico que sostiene el reclamo de los hombres de campo. Esta noción está ligada estrechamente al "país federal" que exigen los productores. Los PMP reivindican "fuertemente" la cuestión federal, en tanto son conscientes que sus problemas están constreñidos a las realidades socioespaciales. Territorio es entonces sinónimo de la dilatada extensión geográfica de ese Interior productivo, y remite, por cierto, a su diversidad intrínseca.

-Lugar: esta categoría es referida al "particularizar" la realidad de los productores, en un nivel geográfico más focal de su problemática, y está directamente ligada con la vulnerabilidad socioeconómica y semiótica que entrañan los lugares más alejados, sin accesibilidad por rutas pavimentadas y confinados como parajes o pueblos pequeños, aunque no por ello menos relevantes desde el punto de vista agropecuario.

-Ruta: constituye una de las categorías más "redundantes" en el mensaje de los productores, ya que se liga a la acción directa y a la semiosis corporal del reclamo de los productores en los últimos tiempos. Por ello, la "ruta" no es sólo una categoría del discurso geográfico, sino que es el significante revelador del problema por su inmanencia intrínseca al "estar ahí" del productor, poniendo su cuerpo en el reclamo.

Publicado en formato digital: Mgtr. Emilas Darlene Carmen Lebus. LA DIALÉCTICA OPRESOR - OPRIMIDO EN LA CONSTRUCCIÓN DISCURSIVA DE LA VULNERABILIDAD DEL PEQUEÑO Y MEDIANO PRODUCTOR AGROPECUARIO DEL NORDESTE ARGENTINO. Revista Geográfica Digital. IGUNNE. Facultad de Humanidades. UNNE. Año 9. No 18. Julio - Diciembre 2012. ISSN 1668-5180 Resistencia, Chaco. En: http://hum.unne.edu.ar/revistas/geoweb/default.htm 
-Distancia: categoría geográfica por excelencia. Es pronunciada para indicar la vulnerabilidad del que vive "alejado" de los puertos de exportación y de los ejes viales estratégicos.

-Circulación: la distancia se "traduce" en los fletes, en la cantidad de cargas realizadas. Si bien, ésta no constituye una categoría precisa, interviene en el análisis geográfico de los flujos, su distribución territorial, su magnitud, sirviendo esto como un medio de conocimiento del problema del transporte en Argentina en relación a los desequilibrios territoriales.

- Ciudad: categoría geográfica que ha emergido con fuerza en el reclamo de los PMP. Aparece en el proceso semiótico cuando la conciencia oprimida deviene en-otro. Este otro es también lo que se opone, la población urbana, especialmente la que vive en las grandes ciudades. El conflicto campo gobierno ha revelado que la ciudad está articulada con la base agropecuaria de nuestra economía, dejando así de ser las antípodas del problema de los productores.

-Campo: categoría poco precisa, ya que, por un lado, alude al espacio donde se desarrollan las actividades agropecuarias y, por otro, refiere a las actividades mismas. Incluso aparece la expresión: "gente del campo", para indicar la realidad "encarnada" por el productor en relación a su medio, convirtiéndose en vivencia, a tal punto que podría afirmarse que el "el campo es su vida, su mundo".

-Rural: calificativo inherente a lo que se da en un medio no urbano. No coincide exactamente con lo agrario, sino más bien con el "espacio", por lo tanto, este término tiene una implicancia geográfica directa. En los discursos aparece expresado como: "trabajo rural", "medio rural". El reclamo de los productores puso de manifiesto un "mundo rural" que existe y tiene caracteres y dinámica propia. Así, lo rural dejó de ser el "gran desconocido" para el hombre de ciudad.

-Urbano: calificativo que designa lo que es concomitante a la población "concentrada" y que realiza actividades no agrarias. Esta noción tiene, como la anterior, un profundo sentido geográfico. En el conflicto campo - gobierno, lo urbano se "descubre" a sí mismo, a la par que comienza a tomar conciencia del otro (de los productores y del mundo rural). De este modo, urbano - rural no aparecen como "opuestos", sino como términos "contradictorios" que se reclaman uno a otro en un proceso de identificación mutua, pues ninguno puede alcanzar su propia esencia si no es pasando por el otro, es decir, por el momento de máxima negatividad (lo que ocurrió en el período de mayor crudeza del conflicto y del corte de rutas en el 2008). Se ha iniciado pues la concienciación, reconociendo sus vínculos a través de las cadenas de valor agroindustriales y al descubrir cómo el problema de los productores termina impactando, no sólo en los pueblos y pequeñas ciudades del Interior, sino -en última instancia- en las grandes ciudades.

Todo esto puso de manifiesto que aquellas categorías espaciales que hasta hace poco tiempo "eran percibidas" como dicotómicas, pasan a constituir los ejes de la dialéctica que sostiene el proceso de concienciación de las situaciones de opresión (y, en consecuencia, de vulnerabilidad) que experimenta el hombre y las comarcas del Interior del país.

\section{CONCLUSIONES "PROVISORIAS"}

El discurso engendra una semiosis y ésta es tan (o más) real que lo que habitualmente llamamos lo real. El discurso "crea" una realidad. Decir esto no implica que estemos postulando un papel omnipresente a la fuerza de la palabra, como si ésta terminara por aniquilar todo ente fuera de ella. En cambio, se busca remarcar que la condición del oprimido sólo se puede manifestar, y a partir de allí, iniciar el camino a la autoconciencia mediante la fuerza de la palabra. Sin embargo, cuando ésta no alcanza para semiotizar una realidad dramática, aparecen otras formas de expresión que permiten al otro "representarse" la realidad que afrontan los sujetos "situados", espacial y socialmente diferenciados en la Geografía del Interior.

Para superar la realidad opresora es preciso salirse, en primer lugar, del temor que cunde al inicio, así como abocarse, mediante una acción comprometida, al develamiento de la verdad. Para conseguirla se requieren ambas cosas; el temor, porque -como dice Hegel- "el miedo al señor es el comienzo de la sabiduría", pero no alcanza con esto. Hace falta también el trabajo formativo, o sea, la acción y perseverancia que posibilita a la conciencia oprimida reencontrarse por sí misma en sentido "propio" (no en un sentido "extraño" como aparenta en principio). Hegel lo sintetiza así:

Para esta reflexión son necesarios los dos momentos, tanto el del temor y el del servicio en general como el de la formación, y ambos, de un modo universal. Sin la disciplina del servicio y la obediencia, el temor se mantiene en lo formal y no se propaga a la realidad consciente de la existencia. Sin la formación, el temor permanece interior y mudo y la conciencia no deviene para ella misma. (ibidem:121)

Publicado en formato digital: Mgtr. Emilas Darlene Carmen Lebus. LA DIALÉCTICA OPRESOR - OPRIMIDO EN LA CONSTRUCCIÓN DISCURSIVA DE LA VULNERABILIDAD DEL PEQUEÑO Y MEDIANO PRODUCTOR AGROPECUARIO DEL NORDESTE ARGENTINO. Revista Geográfica Digital. IGUNNE. Facultad de Humanidades. UNNE. Año 9. No 18. Julio - Diciembre 2012. ISSN 1668-5180 Resistencia, Chaco. En: http://hum.unne.edu.ar/revistas/geoweb/default.htm 
Revista Geográfica Digital. IGUNNE. Facultad de Humanidades. UNNE. Año 9. № 18. Julio - Diciembre 2012. ISSN 1668-5180 Resistencia, Chaco

Sin duda, Hegel está aludiendo al movimiento conceptual, que es un proceso de alumbramiento del sentido "auténtico" sobre algo. Teniendo en cuenta que dicho "movimiento" implica distintos "momentos", podemos decir que la realidad de los PMP, a la luz del conflicto que se ha desatado a partir de la Res. 125, ha permitido -hasta ahora- "suprimir" algunos aspectos del estado inicial (la situación de máxima vulnerabilidad discursiva del PMP), y "conservar" su propia identidad, su saber hacer y su vínculo con el Interior Profundo y con las economías regionales. Falta aún construir el momento de la "superación" de la situación, socioeconómica y semióticamente, que afecta a los PMP, para lo cual es preciso generar una conciencia crítica genuina, que no desnaturalice la esencia del problema (como cuando se niega al otro por el puro hecho de mostrar lo opuesto), sino que, pasando por la negatividad absoluta se pueda retornar a sí mismo, a su ser concreto que es siempre un ser situado, un "singular" (caracterizado por la realidad socioespacial del lugar o comarca donde el PMP realiza su actividad). Sólo así será posible alcanzar el universal, pero retornado del paso por la negatividad, es decir, como "universal concreto". Éste será, a su debido tiempo, el descubrimiento de esa Argentina Profunda que reivindica el PMP, esto es, la singularidad de las regiones productivas del país y la idiosincrasia del hombre del Interior, tan necesarias como el escenario y los actores de la Pampa Húmeda. Esto permitirá concretar el Aufhebung hegeliano (movimiento que suprime, conserva $y$ "supera"), en un contexto de igualdad de oportunidades y mayor reconocimiento al productor radicado en la vasta y diversa Geografía del Interior. Si esto parece lejano, utópico, no lo es el proceso que se ha iniciado. Esto que se ha iniciado es lo más concreto que existe y ello es ya una forma de liberarse de la vulnerabilidad semiótica extrema que aún padecen muchos PMP y que se legitima y, por ende, se "reproduce" cuando el otro (la sociedad, el gobierno) ignora su existencia.

Como bien lo ha expresado Lacan (1968/1969), no todo lo que hay en el mundo deviene en un hecho, sino sólo aquello que se articula en torno al significante. En consecuencia, todo acaecimiento se transforma en hecho en la medida en que es dicho, o mejor aún, "semiotizado". De alguna manera, esto ya lo había anticipado Hegel, desde un punto de vista lógico-semántico, al sostener que no basta con que uno sostenga una idea; ies preciso que lo diga!, porque al decir, se da cuenta que está expresando lo contrario de lo que quería señalar. Esto se ve reflejado en el asombro de los propios productores, cuando al exponer su problema afloraron otras cuestiones de fondo, como la desigualdad, el desequilibrio territorial, el estado crítico de las economías regionales, el unitarismo como filosofía pragmática del ejercicio gubernamental, los acuerdos tácitos al margen de la Constitución, la ausencia de un "proyecto" federal y los desaciertos de la política económica y de la gestión en general frente a los problemas. Por el contrario, se busca acallar la pluralidad de voces con el fin de imponer un discurso único. Esto puede corroborarse analizando los hechos a los que el gobierno otorga significancia, tal como el deseo exasperado de concreción del des-financiamiento de los medios de comunicación críticos, mediante la ejecución de la Ley de Medios. La presión ejercida a los jueces constituye un indicador que confirma la regla. $\mathrm{Y}$, aunque los reclamos de la gente del campo hoy no tienen la misma fuerza que en el 2008, ese fenómeno semiótico singular puso el germen del proceso de concienciación. El camino, pues, está abierto.

\section{BIBLIOGRAFÍA}

-Freire, Paulo. (2008). Pedagogía del Oprimido. Buenos Aires. Siglo Veintiuno.

-Hegel, G.W.F. (2002). Fenomenología del Espíritu. México. Fondo de Cultura Económica.

-Lacan, Jacques. (1968-1969). De un Otro al otro. Seminario XVI: clases dadas por Lacan entre noviembre de 1968 y junio de 1969. Versión de Internet. Material de circulación interna del Doctorado en Ciencias Cognitivas de la Universidad Nacional del Nordeste.

-López, Marta. (2007). "Los bordes de la semiótica y la praxis humana". Actas del VII Congreso Nacional y II Congreso Internacional de la Asociación Argentina de Semiótica. Rosario. En: www.centro-de-semiótica.com.ar/TEXTOS FINALES.html.

-Magariños de Morentín, Juan. (2008). La semiótica de los bordes. Apuntes de metodología semiótica. Córdoba. Ed. Comunicarte.

-Watzlawick, Paul. (1994). ¿Es real la realidad? Barcelona. Herder.

Publicado en formato digital: Mgtr. Emilas Darlene Carmen Lebus. LA DIALÉCTICA OPRESOR - OPRIMIDO EN LA CONSTRUCCIÓN DISCURSIVA DE LA VULNERABILIDAD DEL PEQUEÑO Y MEDIANO PRODUCTOR AGROPECUARIO DEL NORDESTE ARGENTINO. Revista Geográfica Digital. IGUNNE. Facultad de Humanidades. UNNE. Año 9. No 18. Julio - Diciembre 2012. ISSN 1668-5180 Resistencia, Chaco. En: http://hum.unne.edu.ar/revistas/geoweb/default.htm 